\title{
The combination of systemic chemotherapy and local treatment may improve the survival of patients with unresectable metastatic colorectal cancer
}

\author{
JIEMIN ZHAO, LIANGRONG SHI, MEI JI, JUN WU and CHANGPING WU \\ Department of Oncology, The Third Affiliated Hospital of Soochow University, Changzhou, Jiangsu 213000, P.R. China
}

Received January 17, 2017; Accepted March 8, 2017

DOI: $10.3892 / \mathrm{mco} .2017 .1247$

\begin{abstract}
With the development of systemic chemotherapy, the survival time of patients with advanced colorectal cancer (CRC) has increased. In addition, local treatments, such as microwave ablation and radioactive seed implantation, have been shown to be effective. However, the number of studies reporting on the effect of systemic chemotherapy combined with local treatments is limited. The present study was conducted to determine the effect of local treatment combined with systemic chemotherapy in patients with initial unresectable metastatic CRC (mCRC). Clinicopathological and follow-up data from 273 patients with initial unresectable mCRC between April, 2007 and October, 2013 were retrospectively analyzed. A total of 51 patients received minimally invasive treatments combined with systemic chemotherapy and 39 patients achieved tumor-free survival (TFS). The median TFS time was 9 months (range, 2-45 months); the median overall survival (OS) time was 40 months (range, 12-108 months). In patients who did not achieve TFS, the OS was 37 months. Thus, patients who achieved TFS exhibited a significantly longer OS compared with those who did not achieve TFS $(\mathrm{P}=0.049)$. The results of the univariate analysis demonstrated that certain characteristics, such as the number of lesions and maximum tumor diameter, were associated with the achievement of TFS. The patients assessed herein achieved TFS in response to local treatments combined with systemic chemotherapy. Furthermore, the achieved TFS provided an OS benefit.
\end{abstract}

\section{Introduction}

Recent improvements in living standards, changes in dietary habits and ingredients and the increase in the aging population have been associated with increases in the incidence and mortality of colorectal cancer (CRC). Indeed, distant metastases

Correspondence to: Dr Changping Wu, Department of Oncology, The Third Affiliated Hospital of Soochow University, 185 Juqian Street, Changzhou, Jiangsu 213000, P.R. China

E-mail: zoezoe210328010@126.com

Key words: unresectable metastatic colorectal cancer, systemic chemotherapy, local treatment, tumor-free survival, overall survival may be detected in a proportion of newly diagnosed patients, and recurrent or distant metastases have been observed despite radical surgery or postoperative adjuvant therapy. The overall survival (OS) of patients with metastatic CRC (mCRC) is improved by resection of the metastases, with 5-year survival rates of up to $50 \%$ following surgery (1), whereas the survival rates remain low $(<5 \%)$ among patients with unresectable mCRC (2). However, the majority of $\mathrm{mCRC}$ patients are considered to be unresectable at diagnosis (3). Although previous studies have demonstrated that preoperative chemotherapy, or chemotherapy combined with targeted agents, may convert initially unresectable CRC metastases to resectable $(3,4)$, certain patients remain unresectable due to extensive extrahepatic disease or insufficient remaining liver parenchyma or multi-organ metastases.

For unresectable mCRC patients, a combination of chemotherapy and a molecular-targeted agent, either antiangiogenic (i.e., bevacizumab) or a monoclonal antibody epidermal growth factor receptor inhibitor (e.g., cetuximab and panitumumab), is currently considered to be the standard therapy. The CALGB 80405 trial enrolled $>1,100$ wild-type KRAS cases who were randomly assigned to group chemotherapy (either FOLFOX or FOLFIRI) plus either cetuximab or bevacizumab. In the all-RAS wild-type population, the median progression-free survival (PFS) was 11 months in both arms [hazard ratio $(\mathrm{HR})=1.1 ; \mathrm{P}=0.31$, with a median $\mathrm{OS}$ of $31-32$ months $(\mathrm{HR}=0.9 ; \mathrm{P}=0.40)(5)$, which represented a significant improvement. However, in China, these targeted drugs are not included in the health insurance directory, and a proportion of the patients harbor mutations in the RAS or BRAF genes, affecting the application and efficacy of these treatments, as the OS was only 16-22 months for either FOLFOX or FOLFIRI without combination with a molecular-targeted agent (6). Consequently, the rational use of limited resources and the appropriate combination of different treatment modalities to reduce treatment cost, improve the quality of life and prolong survival, is a major focus among Chinese oncologists. Recent oncology studies have reported satisfactory results with local therapy, such as microwave ablation, radioactive seed implantation and intensity-modulated conformal radiotherapy. However, only a limited number of studies have addressed the optimal administration of systemic chemotherapy combined with these local treatments.

The aim of the present retrospective study was to investigate the strategy of the combination of systemic chemotherapy 
with local treatments to improve the OS of patients with initial unresectable $\mathrm{mCRC}$ without using molecular-targeted agents.

\section{Patients and methods}

Patient selection. Between April, 2007 and October, 2013, 273 consecutive patients with biopsy-proven stage IV CRC were treated at the Third Affiliated Hospital of Soochow University (Changzhou, China). Patients who only received systemic chemotherapy or local treatments, or received molecular-targeted agents, were excluded. Patients who exhibited progressive disease (PD) as defined by the Response Evaluation Criteria in Solid Tumors (RECIST 1.1) (7) after two cycles of chemotherapy were also excluded. Finally, 51 patients who received systemic chemotherapy and local treatment were retrospectively analyzed. The patients were aged 35-75 years, had a World Health Organization performance status of $\leq 2$, and were no longer considered candidates for surgery. The characteristics of the patients are summarized in Table I.

This retrospective study was conducted in accordance with the Declaration of Helsinki including all amendments, and was approved by the Independent Research Ethics Committee of the Third Affiliated Hospital of Soochow University. Written informed consent was obtained from all the patients prior to treatment.

Overall treatment principal. Systemic chemotherapy was applied as the initial treatment. For patients with several $(>3)$ or larger $(>5 \mathrm{~cm})$ liver metastases, continuous infusion of floxuridine (FUDR) into the hepatic artery was added. Two or three cycles after the initial chemotherapy, the results of the enhanced spiral-computed tomography (CT) were reviewed. If the disease was controlled and the primary tumor was resectable, surgical removal of the primary tumor was recommended. If the metastatic tumors were reduced to a size of $<5 \mathrm{~cm}$, microwave or radiofrequency ablation were first considered. If the metastases were not suitable for ablation, radioactive seed implantation was recommended. In general, external radiation therapy was administered for pelvic, bone and brain metastases. Once the patients achieved tumor-free survival (TFS), an additional four or six cycles of the original chemotherapy regimen were administered to solidify the effect. Follow-up, including a CT scan and assessment of tumor marker levels, was performed every 2-3 months up to the time of progression. If the patients did not achieve TFS, systemic chemotherapy was administered.

Systemic chemotherapy. A total of 40 patients received an intravenous infusion of 5-fluorouracil (5-FU) and leucovorin with oxaliplatin (FOLFOX) as first-line treatment, 8 patients received capecitabine with oxaliplatin (XELOX), 2 patients received irinotecan with capecitabine (XELIRI), and 1 patient received an intravenous infusion of 5-FU and leucovorin with irinotecan (FOLFIRI). The FOLFOX regimen included i.v. leucovorin calcium at a dose of $200 \mathrm{mg} / \mathrm{m}^{2}$, bolus 5-FU at a dose of $400 \mathrm{mg} / \mathrm{m}^{2}$ and continuous i.v. 5-FU at a dose of $600 \mathrm{mg} / \mathrm{m}^{2}$ on day 1 , followed by $85 \mathrm{mg} / \mathrm{m}^{2}$ oxaliplatin on day 2 . The cycle was repeated every 2 weeks. XELOX consisted of a 2-h intravenous infusion of oxaliplatin $130 \mathrm{mg} / \mathrm{m}^{2}$ on day 1 plus oral administration of capecitabine $1,000 \mathrm{mg} / \mathrm{m}^{2}$ twice daily for 14 days and was repeated every 21 days. XELIRI consisted of a 2-h intravenous infusion of irinotecan $180 \mathrm{mg} / \mathrm{m}^{2}$ on day 1 plus oral administration of capecitabine $1,000 \mathrm{mg} / \mathrm{m}^{2}$ twice daily for 14 days, and was repeated every 21 days. FOLFIRI included i.v. leucovorin calcium at a dose of $200 \mathrm{mg} / \mathrm{m}^{2}$, bolus 5-FU at a dose of $400 \mathrm{mg} / \mathrm{m}^{2}$ and continuous i.v. 5-FU at a dose of $600 \mathrm{mg} / \mathrm{m}^{2}$ on day 1 , followed by $180 \mathrm{mg} / \mathrm{m}^{2}$ irinotecan on day 2 , and was repeated every 2 weeks. A total of 29 patients received FOLFIRI as a second-line regimen, 3 patients received FOLFOX, 3 patients did not undergo second-line chemotherapy as they succumbed to the disease, and second-line chemotherapy was not administered to 16 patients as they did not exhibit disease progression after first-line chemotherapy and local treatments at the last follow-up visit.

Local treatment. A total of 17 patients with unresectable liver metastases underwent implantation using a hepatic artery kit and received hepatic arterial infusion (HAI) therapy. FUDR was delivered via a 14-day infusion at $0.15 \mathrm{mg} / \mathrm{kg} / \mathrm{day}$, and dexamethasone (DXM) was delivered at $1 \mathrm{mg} / \mathrm{m}^{2} /$ day using a pump with FUDR heparin and saline. A total of 38 patients received microwave ablation focused on the liver 49 times, the lung 15 times, and the spleen once, if the metastases were sized $<5 \mathrm{~cm}$. A total of 29 patients received radioactive ${ }^{125} \mathrm{I}$ seed implantation, in which the liver was targeted 14 times, the lung 20 times, the pelvis 9 times, the adrenal glands 4 times, the retroperitoneal lymph nodes once and the abdomen once, when the metastases were not suitable for microwave ablation. A total of 12 patients received external radiation therapy focused on the pelvis 7 times, the bone 4 times, the brain 3 times and the adrenal glands once. The required instruments, materials and the protocols for local treatments were as described in our previous articles (8-11).

Clinical evaluation and follow-up. According to RECIST 1.1, the response to chemotherapy was classified as complete remission (CR), partial remission (PR), progressive disease (PD), or stable disease (SD). Two or three cycles after the initial chemotherapy, enhanced spiral CT results were reviewed, followed by CT review every three cycles. The final follow-up was conducted on April 1, 2016. Dose adjustments were performed in the event of toxicity and assessed according to the National Cancer Institute-Common Terminology Criteria for Adverse Events (NCI-CTCAE), version 3.0 (https://ctep.cancer.gov/protocoldevelopment/electronic_applications/docs/ctcaev3.pdf). TFS was defined as the time from non-detection of tumors on CT after the treatments, until the first discovery of local tumor recurrence or distant metastasis, or until the date of the last follow-up. Overall survival (OS) was defined as the time from the diagnosis of unresectable mCRC to the date of death due to any cause or the date of the last follow-up.

Statistical analysis. The survival rates were estimated using the life table method. The survival analysis was performed by applying the log-rank test. The associations of different potential predictive factors with TFS were assessed using the Fisher's exact test for categorical variables or the Wilcoxon rank-sum test for continuous variables. The statistical analysis was performed using SPSS software, version 18.0 (SPSS, Inc., Chicago, IL, USA). 
Table I. Patient characteristics $(n=51)$.

\begin{tabular}{lcc}
\hline Characteristics & $\mathrm{n}$ & $(\%)$ \\
\hline Gender & & \\
Male & 40 & $(78.4)$ \\
Female & 11 & $(21.6)$ \\
Median age, years (range) & $62(35-75)$ & \\
Location of primary cancer & & \\
Rectum & 21 & $(41.2)$ \\
Colon & 30 & $(59.8)$ \\
Resection of primary cancer & & \\
Yes & 42 & $(82.4)$ \\
No & 9 & \\
Location of metastasis & & $(17.6)$ \\
Liver & \\
Lungs & 14 & $(13.7)$ \\
$>3$ locations & 7 & \\
Line of chemotherapy & 30 \\
First-line & & \\
Second- and further line & 48 & \\
\hline
\end{tabular}

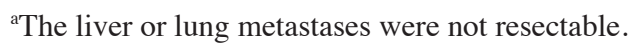

\section{Results}

Remission rates and survival time. According to RECIST 1.1, among the 51 patients who were evaluated, after two or three cycles of initial chemotherapy, 0 patients achieved CR, 36 achieved PR (70.6\%) and 15 achieved SD (29.4\%), with an objective response rate of $100 \%$. Of the 9 patients with newly diagnosed and unresectable primary tumors, 8 underwent primary tumor resection after treatment, and the resection rate was $88.9 \%$. At the last follow-up, 34 of the 51 patients had succumbed to the disease and 17 remained alive. The median OS was 40 months (range, 12-108 months). With the combination of minimally invasive treatments with systemic chemotherapy, 39 patients obtained TFS. Among those 39 patients, the TFS was 2-45 months, and the median TFS was 9 months. The median OS of patients who achieved and those who did not achieve TFS was 40 and 37 months, respectively. The patients who obtained TFS had a significantly better OS compared with those who did not $(\mathrm{P}=0.049$, Fig. 1$)$. The results of the univariate analysis demonstrated that certain characteristics, such as the number of lesions and maximum tumor diameter, were associated with the achievement of TFS (Table II).

Chemotherapy-related toxicity. Grade 3-4 myelosuppression occurred in 32 patients $(63.2 \%)$, mainly manifesting as neutropenia. Grade 1-2 liver toxicity developed in 37 patients (72.4\%), grade 1-2 neurotoxicity occurred in 10 patients $(19.7 \%)$, whereas no allergic reactions were observed as a consequence of oxaliplatin. Grade 1-2 nausea or vomiting was documented in 27 patients $(53.9 \%)$. Of the 17 patients who underwent a procedure with a hepatic artery kit with continuous FUDR perfusion, 7 (41.2\%) experienced grade 3-4 diarrhea, $15(88.2 \%)$

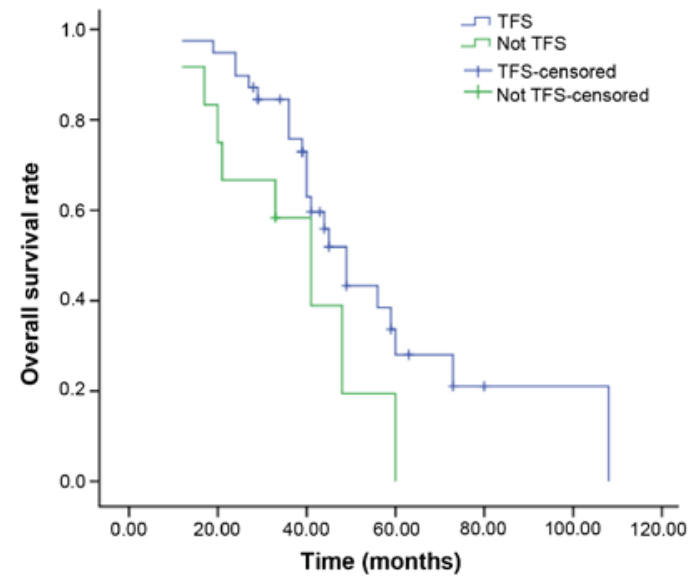

Figure 1. Overall survival of the patients who achieved and those who did not achieve TFS after systemic chemotherapy and local treatments. TFS, tumor-free survival.

had grade 1-2 abdominal pain and 2 (11.8\%) experienced a local infection and required debridement. All the side effects were restored following drug withdrawal and symptomatic treatment, and no deaths were reported (Table III).

Severe local treatment-related complications were not observed in any of the patients. Common complications included puncture pain, vomiting, fever, bone marrow suppression, transient liver damage and pneumothorax, all of which responded to symptomatic treatment.

Improvement of clinical symptoms. Among the 15 patients experiencing pain, 11 achieved symptom relief; the remission rate was $73.3 \%$. Chest tightness and shortness of breath were improved in 3 patients with lung metastases; the remission rate was $100 \%$. Of the 51 patients, $18(35.3 \%)$ experienced weight gain.

\section{Discussion}

CRC is the third most common malignant tumor worldwide and has the second highest mortality among malignant tumors (12). Metastasis occurs in $\sim 30 \%$ patients after the initial diagnosis. Systemic chemotherapy is the principal therapeutic modality in patients with unresectable mCRC. In recent years, palliative chemotherapy for unresectable $\mathrm{mCRC}$ has progressed considerably, particularly with the application of irinotecan and oxaliplatin, resulting in an increase in the median survival time from 16 to 22 months (6). In addition, the application of targeted drugs has further improved patient survival (13). However, targeted drugs are still not widely used in China, and RAS or BRAF mutations in patients limit the application and efficacy of molecular-targeted treatments. Thus, there is a need for better understanding the variety of available treatment modalities in order to improve survival and quality of life without significantly increasing the total treatment cost. However, strategies to achieve such goals remain underinvestigated.

Local treatments, such as microwave ablation and radioactive seed implantation, have been shown to be effective $(14,15)$.

In the present study, for patients with several $(>3)$ or larger $(>5 \mathrm{~cm}$ ) liver metastases, continuous infusion of FUDR into the 
Table II. Univariate analysis of the predictors of TFS.

\begin{tabular}{|c|c|c|c|}
\hline Characteristics & TFS $(n=39)$ & No TFS $(n=12)$ & P-value \\
\hline Gender & & & 0.262 \\
\hline Male & 32 & 8 & \\
\hline Female & 7 & 4 & \\
\hline $\begin{array}{l}\text { Site of primary } \\
\text { cancer }\end{array}$ & & & 0.739 \\
\hline Colon & 22 & 8 & \\
\hline Rectum & 17 & 4 & \\
\hline $\begin{array}{l}\text { Location of } \\
\text { metastasis }\end{array}$ & & & 0.134 \\
\hline Liver & 13 & 1 & \\
\hline Lungs & 6 & 1 & \\
\hline >3 locations & 20 & 10 & \\
\hline Number of lesions & & & 0.002 \\
\hline$\leq 5$ & 28 & 2 & \\
\hline$>5$ & 11 & 10 & \\
\hline $\begin{array}{l}\text { Maximum diameter } \\
\text { of the tumor, } \mathrm{cm}\end{array}$ & & & 0.001 \\
\hline$\leq 5$ & 33 & 5 & \\
\hline$>5$ & 6 & 7 & \\
\hline \multicolumn{4}{|l|}{ Age, years } \\
\hline Median & 63 & 60 & 0.820 \\
\hline Range & $42-75$ & $35-78$ & \\
\hline
\end{tabular}

TFS, tumor-free survival.

Table III. Frequency of chemotherapy-related toxicities.

\begin{tabular}{lcl}
\hline Toxicities & $\mathrm{n}$ & $(\%)$ \\
\hline Myelosuppression & 32 & $(63.2)$ \\
Liver damage & 37 & $(72.4)$ \\
Neuropathy & 10 & $(19.7)$ \\
Diarrhea & 7 & $(41.2)$ \\
Allergy & 0 & $(0.0)$ \\
Anorexia/nausea & 27 & $(53.9)$ \\
Fatigue & 6 & $(11.8)$ \\
Hand-foot syndrome & 2 & $(3.9)$ \\
Other & 2 & $(3.9)$ \\
Any grade $\geq 3$ & 9 & $(17.6)$ \\
\hline
\end{tabular}

hepatic artery was added. When doxifluridine was infused into the hepatic artery, the drug uptake rate by the liver was $95 \%$, and the drug concentration in the tumors was 16 times higher compared with that assessed using conventional intravenous chemotherapy (16). Continuous infusion using a hepatic arterial kit may be effective in maintaining drug concentrations and extending the delivery time, thereby increasing the drug sensitivity. Shi et al (11) reported that CRC patients with liver metastasis who were treated with HAI FUDR and systemic XELOX experienced a high resection rate for asymptomatic
$\mathrm{CRC}$ with unresectable liver metastases, as well as a low rate of complications associated with unresectable primary cancer.

Metastasis resection may significantly improve the prognosis of patients with mCRC; however, certain challenges remain for patients with unresectable metastasis but no symptoms from the primary lesions. Controversial retrospective studies have been conducted to determine whether it is appropriate to resect the primary tumor in patients with mCRC (17). In the present study, if the disease was controlled and the primary tumor was considered to be resectable, it was recommended that patients undergo resection of the primary tumor. Systemic chemotherapy combined with resection of the primary tumor provided a proportion of the patients in this group with TFS and improved their quality of life. Thus, it is crucial for clinicians to identify the appropriate opportunities for this treatment.

Following systemic chemotherapy, the metastatic tumors regressed in a proportion of the patients. A number of clinical studies $(18,19)$ support the use of capecitabine as maintenance therapy, as it has been shown to prolong PFS, but provides no OS benefit. It is recommended that such patients receive a local, minimally invasive treatment to achieve TFS. Some residual cancer cells may persist after complete radiographic remission. After a few cycles of chemotherapy, these remaining tumor cells may become resistant to chemotherapeutic drugs; they may not only become resistant to the same type of drug, but may also develop cross-resistance to other, previously unused drugs. Thus, the effect may not be optimal, even in the presence of other chemotherapeutic drugs. In addition, several cycles of chemotherapy may impair the immune system of the patient and result in unendurable toxic adverse effects. These phenomena all promote tumor progression and, in such cases, chemotherapy was terminated and other methods were used to achieve TFS, such as microwave or radiofrequency ablation, radioactive seed implantation, or intensity-modulated conformal radiotherapy, depending on the locations of the metastases in each patient. Petre et al (20) used radiofrequency ablation to treat 45 patients with 69 lung metastases resulting from CRC. The 3-year local control rate was $89 \%$, and the 1-, 2- and 3-year OS rates were 95, 72 and $50 \%$, respectively. Zhou et al (21) reported that microwave treatment may also enhance local and systemic immune function. Wang et al (22) reported a local control rate of $87 \%$ using CT-guided ${ }^{125}$ I seed implantation to treat recurrent colorectal cancer, with 1- and 2-year survival rates of 93 and 50\%, respectively. In the present study, 29 patients underwent radioactive ${ }^{125} \mathrm{I}$ seed implantation, including 14 times in the liver, 20 times in the lung, 9 times in the pelvis, 4 times in the adrenal glands, once in the retroperitoneal lymph nodes and once in the abdominal wall. Compared with other treatment methods, radioactive ${ }^{125} \mathrm{I}$ seed implantation was simple, had a reduced impact on the surrounding normal tissue, was reliable and resulted in fewer complications, particularly in patients with local recurrence or metastases in the adrenal glands, retroperitoneal lymph nodes and abdominal wall. Furthermore, this approach effectively controlled local recurrence and tumor growth and improved patient survival. Of note, the successful implementation of this technique depends on the experience of the surgeon. Our sample included a 75-year-old male patient with lung metastasis after ascending colon resection: The lung metastasis was isolated, but due to the poor cardiopulmonary function of the patient, surgical 
resection was not possible. The patient received initial systemic chemotherapy and radioactive ${ }^{125}$ I seed implantation, and there were no new metastases after $>3$ years of follow-up. The patient experienced a significant improvement in his quality of life and prolonged survival. In the present study, certain characteristics, such as the number of lesions and the maximum diameter of the tumor, were associated with patients who achieved TFS. However, if local treatment was successfully performed, its effects were also associated with those characteristics.

The OPTIMOX1 study (23) demonstrated that six cycles of oxaliplatin using a simplified LV/5-FU solution to maintain the chemotherapeutic effect exerted the same effect on the response rate, PFS and OS, compared with continuous use of oxaliplatin until the patients could no longer tolerate the side effects or experienced disease progression; furthermore, with this treatment, patients were able to avoid grade 3-4 toxicities after six cycles. In centers in which $>50 \%$ of the patients received a second treatment with the FOLFOX7 program, the OS was $>25$ months. These results indicate that intense chemotherapy after maintenance therapy may prolong the survival of patients. We also observed that oxaliplatin was effective after a period of inactivity. In the present study, the majority of the patients tolerated FOLFOX or XELOX and FOLFIRI as first-line and second-line chemotherapy, respectively. When the patients achieved TFS, the chemotherapeutic regimen was suspended until the tumors relapsed. Thus, the majority of the patients tolerated the applied treatments, including oxaliplatin as third-line therapy, which was effective in some patients and may prolong OS.

In summary, the results of the present study indicate that, in patients with unresectable $\mathrm{mCRC}$ who are not suitable to receive molecular-targeted agents, particularly patients with $<5$ metastatic lesions or a maximum tumor diameter of $<5 \mathrm{~cm}$, systemic chemotherapy may be administered combined with local treatment to achieve TFS, and the TFS benefits are subsequently converted to a prolongation of OS. To the best of our knowledge, this strategy has not been previously reported. However, this was a retrospective study, and the analyzed cohort was small. Additionally, there were some indications for minimally invasive treatments, resulting in a selection bias. Thus, future prospective randomized studies are required to confirm our results. The use of drugs such as capecitabine as maintenance therapy or combined with molecular-targeted agents to prolong TFS and OS should also be assessed in future investigations.

\section{References}

1. Mayo SC and Pawlik TM: Current management of colorectal hepatic metastasis. Expert Rev Gastroenterol Hepatol 3: 131-144, 2009.

2. Ye LC, Liu TS, Ren L, Wei Y, Zhu DX, Zai SY, Ye QH, Yu Y, $\mathrm{Xu}$ B, Qin XY and Xu J: Randomized controlled trial of cetuximab plus chemotherapy for patients with KRAS wild- type unresectable colorectal liver-limited metastases. J Clin Oncol 31: 1931-1938, 2013.

3. Morris EJ, Forman D, Thomas JD, Quirke P, Taylor EF, Fairley L, Cottier B and Poston G: Surgical management and outcomes of colorectal cancer liver metastases. Br J Surg 97: 1110-1118, 2010.

4. Folprecht G, Gruenberger T, Bechstein W, Raab HR, Weitz J, Lordick F, Hartmann JT, Stoehlmacher-Williams J, Lang H, Trarbach T, et al: Survival of patients with initially unresectable colorectal liver metastases treated with FOLFOX/cetuximab or FOLFIRI/cetuximab in a multidisciplinary concept (CELIM study). Ann Oncol 25: 1018-1025, 2014.
5. Formica $\mathrm{V}$ and Roselli M: Targeted therapy in first line treatment of RAS wild type colorectal cancer. World J Gastroenterol 21: 2871-2874, 2015.

6. Tournigand C, André T, Achille E, Lledo G, Flesh M, Mery-Mignard D, Quinaux E, Couteau C, Buyse M, Ganem G, et al: FOLFIRI followed by FOLFOX6 or the reverse sequence in advanced colorectal cancer: A randomized GERCOR study . J Clin Oncol 22: 229-237, 2004.

7. Eisenhauer EA, Therasse P, Bogaerts J, Schwartz LH, Sargent D, Ford R, Dancey J, Arbuck S, Gwyther S, Mooney M, et al: New response evaluation criteria in solid tumours: Revised RECIST guideline (version 1.1). Eur J Cancer 45: 228-247, 2009.

8. Shi L, Wu C, Wu J, Zhou W, Ji M, Zhang H, Zhao J, Huang Y, Pei H, Li Z, et al: Computed tomography-guided permanent brachytherapy for locoregional recurrent gastric cancer. Radiat Oncol 7: 114, 2012

9. Shi L, Li X, Pei H, Zhao J, Qiang W, Wang J, Xu B, Chen L, Wu J, Ji M, et al: Phase II study of computed tomography-guided (125)I-seed implantation plus chemotherapy for locally recurrent rectal cancer. Radiother Oncol 118: 375-381, 2016.

10. Shi L, Chen L, Wu C, Zhu Y, Xu B, Zheng X, Sun M, Wen W, Dai X, Yang M, et al: PD-1 blockade boosts radiofrequency ablation-elicited adaptive immune responses against tumor. Clin Cancer Res 22: 1173-1184, 2016.

11. Shi L, Zhao J, Lu Q, Chen X, Wang H, Jiang Y, Wu J, Ji M, Xu B, Chen L, et al: Initial hepatic artery infusion and systemic chemotherapy for asymptomatic colorectal cancer with un-resectable liver metastasis. Int J Clin Exp Med 8: 1000-1008, 2015.

12. Jemal A, Siegel R, Ward E, Hao Y, Xu J, Murray T and Thun M: Cancer statistics, 2008. CA Cancer J Clin 58: 71-96, 2008.

13. Skitzki JJ and Chang AE: Hepatic artery chemotherapy for colorectal liver metastases: Technical considerations and review of clinical trials. Surg Oncol 11: 123-135, 2002.

14. Eng OS, Tsang AT, Moore D, Chen C, Narayanan S, Gannon CJ, August DA, Carpizo DR and Melstrom LG: Outcomes of microwave ablation for colorectal cancer liver metastases: A single center experience. J Surg Oncol 111: 410-413, 2015.

15. Wang Z, Lu J, Liu L, Liu T, Chen K, Liu F and Huang G: Clinical application of CT-guided (125)I seed interstitial implantation for local recurrent rectal carcinoma. Radiat Oncol 6: 138, 2011.

16. Van Loon K and Venook AP: Curable patient with metastatic colorectal cancer: Balancing effective therapies and toxicities. J Clin Oncol 32: 991-996, 2014.

17. Venderbosch S, de Wilt JH, Teerenstra S, Loosveld OJ, van Bochove A, Sinnige HA, Creemers GJ, Tesselaar ME, Mol L, Punt CJ and Koopman M: Prognostic value of resection of primary tumor in patients with stage IV colorectal cancer: Retrospective analysis of two randomized studies and a review of the literature. Ann Surg Oncol 18: 3252-3260, 2011.

18. Tournigand C, Samson B, Scheithauer W, Lledo G, Viret, F, Andre T, Ramée JF, Tubiana-Mathieu N, Dauba J, Dupuis O, et al: Bevacizumab (Bev) with or without erlotinib as maintenance therapy, following induction first-line chemotherapy plus Bev, in patients with metastatic colorectal cancer (mCRC): Efficacy and safety results of the international GERCOR DREAM phase III trial. J Clin Oncol 30 (suppl): S3500, 2012.

19. Yalcin S, Uslu R, Dane F, Yilmaz U, Zengin N, Buyukunal E, Buyukberber S, Camci C, Sencan O, Kilickap S, et al: Bevacizumab+ capecitabine as maintenance therapy after initial bevacizumab+ XELOX treatment in previously untreated patients with metastatic colorectal cancer: Phase III 'stop and go' study results-a Turkish oncology group trial. Oncology 85: 328-335, 2013.

20. Petre EN, Jia X, Thornton RH, Sofocleous CT, Alago W, Kemeny NE and Solomon SB: Treatment of pulmonary colorectal metastases by radiof requency ablation. Clin Colorectal Cancer 12: 37-44, 2013.

21. Zhou P, Liang P, Dong B, Yu X, Han Z and Xu Y: Phase I clinical study of combination therapy with microwave ablation and cellular immunotherapy in hepatocellular carcinoma. Cancer Biol Ther 11: 450-456, 2011.

22. Wang J, Yuan H and Liu J: The effects of CT guided interstitial 125I seed implantation in the treatment of recurrent colorectal cancer. J Chin Radiation Oncol 15: 319-322, 2006.

23. Tournigand C, Cervantes A, Figer A, Lledo G, Flesch M, Buyse M, Mineur L, Carola E, Etienne PL, Rivera F, et al: OPTIMOX1: A randomized study of FOLFOX4 or FOLFOX7 with oxaliplatin in a stop-and-Go fashion in advanced colorectal cancer-a GERCOR study. J Clin Oncol 24: 394-400, 2006. 